\title{
Cochrane-Review
}

\section{Scheut der Muskelkater kaltes Wasser?}

\author{
Sport kann schön sein, strengt aber an. Ein Cochrane-Review hat nun \\ untersucht, ob sich die schmerzhaften Folgen ausge- \\ dehnter Leibesübungen mit kaltem Wasser um- \\ gehen lassen. Fazit nach mehr als 120 Seiten: \\ Nichts Genaues weiß man nicht.
}

— Kaltwasserbäder nach dem Training sind ein derzeit beliebtes Mittel, weniger erwünschte Folgen des Sports wie den Muskelkater gar nicht erst aufkommen zu lassen. Ob diese Methode wirkt, ist allerdings nicht klar, wie überhaupt der Muskelkater in Ursache, Vorbeugung und Therapie eines der großen medizinischen Rätsel darstellt.

Im Versuch, etwas mehr Licht in die Turnhallen dieser Welt zu bringen, haben Cochrane-Forscher um Chris Bleakley von der University of Ulster, Newtownabbey, 17 kleine Studien mit zusammen 366 Pro-

banden, die sich dem feuchtkalten Thema gewidmet hatten, aus diversen Datenbanken ausgegraben. Mit sportlicher Ausdauer analysierten die Forscher die Ergebnisse auf 127 Seiten. Doch im Zieleinlauf war klar: Trotz einiger Hinweise auf positive Effekte kalter Tauchbäder wird die Prävention des Leidens, für das schon einmal der Terminus „akute Überlastungsmyopathie" vorgeschlagen wurde, für die Sportmedizin auch künftig rätselhaft bleiben.

Katerprävention

\section{Muntermacher für müde Muskeln}

Kaltes Wasser kann, wie oben beschrieben, dem Muskelkater vermutlich vorbeugen. Das Verfahren stammt aus dem Spitzensport, wird aber auch von Freizeitsportlern gern angewendet. Ursprünglich dienten kalte Bäder der akuten Therapie von Verstauchungen, Zerrungen und Prellungen. Von dem eisigen Nass verspricht man sich antientzündliche Effekte und eine Flüssigkeitsverlagerung von intrazellulär nach intravaskulär, der kardiale Output und der Blutfluss sollen erhöht, Nährstoffe herbei- und Metaboliten abtransportiert werden - vom psychologischen Effekt zu schweigen.

Nichtstun jedenfalls lässt den Muskelkater schnurren, das hat der Cochrane-Review gezeigt. Für Aussagen über andere Verfahren reichten die Daten nicht aus. Mit Kaltwasserbädern konkurrieren in der Katerprävention nach starken Belastungen beispielsweise

- Wechselbäder: Das Ziel ist auch hier eine verbesserte Durchblutung.
- Warmwasserbäder: Die nasse Prävention für Zimperliche.

- Aktive Erholung: Mäßige Bewegung, etwa 15-minütiges leichtes Jogging, soll die Durchblutung steigern - und diese wiederum Metaboliten wie Laktat ausschwemmen.

- Kompression: Kompressionsstrümpfe erfreuen sich vor allem bei Läufern einiger Beliebtheit. Die Idee dahinter ist, mit dem verengten Venendurchmesser die Strömungsgeschwindigkeit des Blutes zu erhöhen, wodurch Gewebeflüssigkeit leichter ausgeschwemmt und die Regenration erleichtert werden soll.

Notabene: Wissenschaftlich nachgewiesen sind diese Wirkungen allesamt nicht. Nur ein Rezept ist sicher, wie der Berliner Sportmediziner Prof. Dieter Böning weiß: „Die beste Vorbeugung gegen Muskelkater ist, inn für eine bestimmte Bewegungsform bereits erlitten zu haben"

$\mathrm{RB}$ =
Die Kaltwasserbäder in den einzelnen Untersuchungen dauerten zwischen drei und 24 Minuten, bei

Wassertemperaturen von frischen fünf bis $15^{\circ} \mathrm{C}$. Vorangegangen waren ihnen verschiedene Arten sportlicher Bewegung, von Jogging über Radfahren hin zu Sportarten, die es nur im Labor gibt, wie 100-faches Repetieren der Bewegung einzelner Muskelgruppen. Für die Muskelschmerzen nach 24,48 und 72 Stunden ergaben sich Unterschiede, bezogen auf eine $10 \mathrm{~cm}$ lange visuelle Analogskala, von -1,27 $\mathrm{cm},-1,58 \mathrm{~cm}$ bzw. $-2,16 \mathrm{~cm}$ zugunsten des Kaltwasserbadens im Vergleich zum Verzicht darauf. Hierbei gilt eine Differenz von $1,4 \mathrm{~cm}$ als minimaler klinisch relevanter Unterschied.

„Wir haben einige Anhaltspunkte dafür gefunden, dass Kaltwasserbäder verglichen mit Nichtstun den verzögerten Muskelschmerz nach körperlichem Training reduzieren“, schreiben die Cochrane-Analytiker um Bleakley. Für einen Vergleich mit anderen Interventionen, etwa Kompressionsbehandlung, aktive Erholung und Wechsel- oder Warmwasserbäder, habe die Datenbasis nicht ausgereicht - wie überhaupt die vorgefundenen Studien nicht der obersten Wissenschaftsliga zuzurechnen seien. Vor allem hätten die meisten Untersuchungen nicht auf etwaige Nebenwirkungen der eisigen Prävention geachtet.

DR. ROBERT BUBLAK .

- Bleakley C et al. Cold-water immersion cryotherapy for preventing and treating muscle soreness after exercise. Cochrane Database of Systematic Reviews 2012, Issue 2 http://doi. wiley.com/10.1002/14651858.CD008262.pub2 